\title{
BrainTV: a novel approach for online mapping of human brain functions
}

\author{
JEAN-PHILIPPE LACHAUX, KARIM JERBI ${ }^{1}$, OLIVIER BERTRAND ${ }^{1}$, \\ LORELLA MINOTTI $^{2}$, DOMINIQUE HOFFMANN ${ }^{3}$, BENJAMIN SCHOENDORFF ${ }^{1}$, \\ and PHILIPPE KAHANE ${ }^{2}$
}

\author{
${ }^{1}$ INSERM, U821, Lyon, F-69500, France; Institut Fédératif des Neurosciences, Lyon, F-69000, France; \\ Université Lyon 1, Lyon, F-69000, France. \\ 2 Department of Neurology and INSERM U704, Grenoble Hospital, Grenoble, France. \\ ${ }^{3}$ Department of Neurosurgery and INSERM U318, Grenoble Hospital, Grenoble, France.
}

\begin{abstract}
Our understanding of the brain's functional organisation has greatly benefited from occasional exploratory sessions during electrophysiological studies, trying various manipulations of an animal's environment to trigger responses in particular neurons. Famous examples of such exploration have unveiled various unexpected response properties, such as those of mirror neurons. This approach, which relies on the possibility to test online the reactivity of precise neural populations has no equivalent so far in humans. The present study proposes and applies a radically novel framework for mapping human brain functions in ecological situations based on a combination of a) exploratory sessions, using real-time electrophysiology to formulate hypotheses about the functional role of precise cortical regions and b) controlled experimental protocols specifically adapted to test these hypotheses. Using this two-stage approach with an epileptic patient candidate for surgery and implanted with intracerebral electrodes, we were able to precisely map high-level auditory functions in the patients' superior temporal lobe. We propose that this procedure constitutes at the least a useful complement of electrical cortical stimulations to map eloquent brain areas in epileptic patients before their surgery, but also a path of discovery for human functional brain mapping.
\end{abstract}

Key terms: speech, gamma rhythm, beta rhythm, brain computer interface, neurofeedback, neural synchronization.

\section{INTRODUCTION}

For patients with intractable epilepsy who require brain surgery, the success and safety of the procedure crucially depends on the correct definition of the brain volume to be resected. This requires not only the identification of the epileptogenic network but also a precise understanding of the functional organisation of the cortex in which it is embedded, so that eloquent cortical regions can be spared (Lesser et al., 1998). Due to large interindividual variations in functional cortical organisation (Ojemann et al., 1989), especially when lesions are present, precise mapping between cortical structures and functions must be performed for each individual patient, and cannot be based on the sole functional neuroimaging literature.

To date, most clinical epilepsy centres rely on electric cortical stimulations (ECS) to reveal the functions of implanted cortical areas, due to the direct effect electric cortical stimulations have on the underlying processes carried by the neural population stimulated (Lesser et al., 1998). This procedure has proved very effective in mapping several functional systems, such as the motor system, the vestibular system or several aspects of the language system, among others (Kahane et al., 2003, Lesser

Correspondence should be addressed to: Jean-Philippe Lachaux (lachaux@lyon.inserm.fr) Mental Processes and Brain Activation INSERM - Unité 280, Centre Hospitalier Le Vinatier, Bâtiment 452, 95 Boulevard Pinel, 69500 BRON, FRANCE, Phone : 33472138900 , Fax : 33472138901 
et al., 1998, Lobel et al., 2001, Luders et al., 1995, Ojemann et al., 1989, Penfield and Jasper, 1954). However it may not be best suited to exploring complex cognitive functions which are unlikely to be active at the time of stimulation, such as mental calculation for instance.

Another approach is to engage patients in cognitive protocols involving the corresponding systems and test for associated modulations in the signal of the implanted electrodes (Lachaux et al., 2003). In particular, it has been suggested recently that task-induced energy modulations of intracranial EEG in the high gamma band $(>70 \mathrm{~Hz})$ constitute an efficient marker for cognitive mapping that can usefully complement the standard ECS approach (Sinai et al., 2005). Indeed, over the last few years, it has become increasingly clear that most cognitive processes elicit, within their underlying cortical networks, highly anatomically and functionally selective gamma band energy modulations. To cite only human intracranial studies, this has been demonstrated for memory (Fell et al., 2001, Howard et al., 2003, Mainy et al., 2007), visual attention and perception, audition (Bidet-Caulet et al., 2003, Crone et al., 2001, Edwards et al., 2005), somatosensory and motor processes (Lachaux et al., 2005, Pfurtscheller et al., 2003, Szurhaj et al., 2005) and language (Crone et al., 2001). These studies expanded on a large field of research on gamma band activity in animals (Singer, 1999) and in human scalp EEG studies (Tallon-Baudry and Bertrand, 1999).

We have used this Dynamic Spectral Imaging (DSI) approach (i.e. the time frequency analysis of intracerebral EEG activity to extract task-induced gamma band modulations) for the past few years, in complement with ECS, and our conclusion is that DSI is very efficient to identify which particular cortical regions are involved in a pre-defined cognitive process, but less efficient to answer the reverse question, that is, which cognitive process is mediated by a pre-defined cortical region. This comes from two limitations of DSI, as it is presently used.

The first limitation comes from the exquisite functional specificity of the gamma band, which leads to the fact that, in our experience, a given cognitive task, such as encoding and maintaining a list of letters into working memory, triggers gamma energy modulations in less than $10 \%$ of all the recorded sites (Mainy et al., 2007). Since the anatomical distribution of those modulations varies with the task, it is theoretically possible to test the patient in a large repertoire of tasks to eventually activate most of the sites and find out their functional specificity. However, this is a long procedure that may prove uncomfortable for the patient.

The second limitation is that this approach can only reveal cognitive processes for which protocols have been tailor-made in advance. The design of a cognitive task is a complex process the success of which depends on a careful choice of control conditions. The number of cognitive processes that may be carried by the recorded sites vastly exceeds the number of protocols that can reasonably be thought of and set up by a single clinical group. Furthermore, there is no way to anticipate all the possible situations that can trigger activity in a particular brain area. As a matter of fact, many important discoveries in electrophysiology came through open exploration, as happened with the discovery of mirror neurons in the frontal lobe of macaque monkeys (di Pellegrino et al., 1992). The use of a set of standard protocols is thus not necessarily best suited for exploration.

To a certain extent, the usefulness of ECS mapping comes from the fact that it is largely exploratory: it relies on the verbal reports of the patient describing the effects produced by the stimulation, or the observation of electrically-elicited behaviours. Those reports can potentially reveal cognitive processes that had not been envisioned beforehand. The objective of this study was to develop an approach based on DSI that would allow for such an exploratory phase. Increments in computing power have fuelled resurgent interest in the application of online analysis of human brain activity. To date, most of the research has focused on one particular application, the transformation of neural measures into 
commands to control an external device (Brain Computer Interface) (Vaughan et al., 2003). However, the range of applications of these techniques is even wider. In particular, they can provide a way to explore the behaviour of neural measures in real-time, while a subject engages into various activities.

The present study proposes and illustrates a functional mapping procedure centred on neurofeedback. We designed a simple computer interface to display in real-time to the patients their activity recorded at particular cortical locations in several frequency bands including the alpha $(8-12 \mathrm{~Hz})$, beta $(12-30 \mathrm{~Hz})$ and gamma bands $(>40 \mathrm{~Hz})$. During such neurofeedback sessions, this device allowed us to identify in an epileptic patient a selectivity for speech in the mid portion of his superior temporal cortex, for sentences or words, but not for short nonsensical speech patterns such as 'patapatapata', or other vocal sounds or the patient's own speech. Those observations were statistically validated in subsequent sessions using experimental paradigms testing specifically those response properties.

\section{METHODS}

\section{Patient and recordings}

The patient suffered from drug-resistant partial epilepsy and was a candidate for surgery. Magnetic resonance imaging of the brain (MRI) showed a left hippocampal sclerosis. Because the location of the epileptic focus could not be identified using non-invasive methods, the patient underwent intracerebral EEG recordings by means of stereotactically implanted multilead depth electrodes (SEEG) (for explanation of this methodology, see (Kahane et al., 2004), on the basis of which the epileptogenic zone proved to be left antero-mesial temporal. Selection of sites to implant was solely based on clinical considerations with no reference to the present experimental protocol. The patient performed the experimental task 11 days after implantation of the electrodes, and had previously given his informed consent to participate in the experiment.

Twelve semi-rigid electrodes were implanted in cortical areas related to the suspected origin of the seizures. Each electrode had a diameter of $0.8 \mathrm{~mm}$ (shown on figure 1) and comprised $5,10,15$ or 18 $2 \mathrm{mms}$ long leads, $1.5 \mathrm{~mm}$ apart (Alcys, Besançon, France), depending on the target region. The electrode contacts were identified on the patient's individual stereotactic scheme, and then anatomically localized using Talairach and Tournoux's proportional atlas (Talairach and Tournoux, 1988). In addition, computer-assisted matching of post-implantation CT-scan with a pre-implantation 3-D MRI afforded a direct visualization of the electrode contacts with respect to the patient's brain anatomy (Voxim, Germany). Intracerebral recordings were obtained using an audio-video-EEG monitoring system (Micromed, Treviso, Italy), allowing for the simultaneous recording of 128 depth-EEG channels sampled at $512 \mathrm{~Hz}$ [0.1-200 Hz bandwidth].

Intracerebral electrical stimulations were performed for purely clinical purposes over a few days, during sessions that lasted 1 to 2 hours and under video-SEEG control. Following our standard clinical practice (Kahane et al., 1993), stimulations at $1 \mathrm{~Hz}$ (3 $\mathrm{mA})$ and $50 \mathrm{~Hz}(1-3 \mathrm{~mA})$ were applied between contiguous contacts at different levels along the axis of all the electrodes. Bipolar stimuli were delivered using a constant current rectangular pulse generator designed for a safe diagnostic stimulation of the human brain (Micromed, Treviso, Italy), according to parameters proven to produce no structural damage.

\section{PARADIGM}

\section{Neurofeedback session}

The neurofeedback consisted of presenting the patient with his imaged cerebral activity in four cortical sites at a time, appearing as curves displaying energy levels measured in several frequency bands over the previous ten seconds (Figure 1). This simple device allowed the patient easily to focus on one 

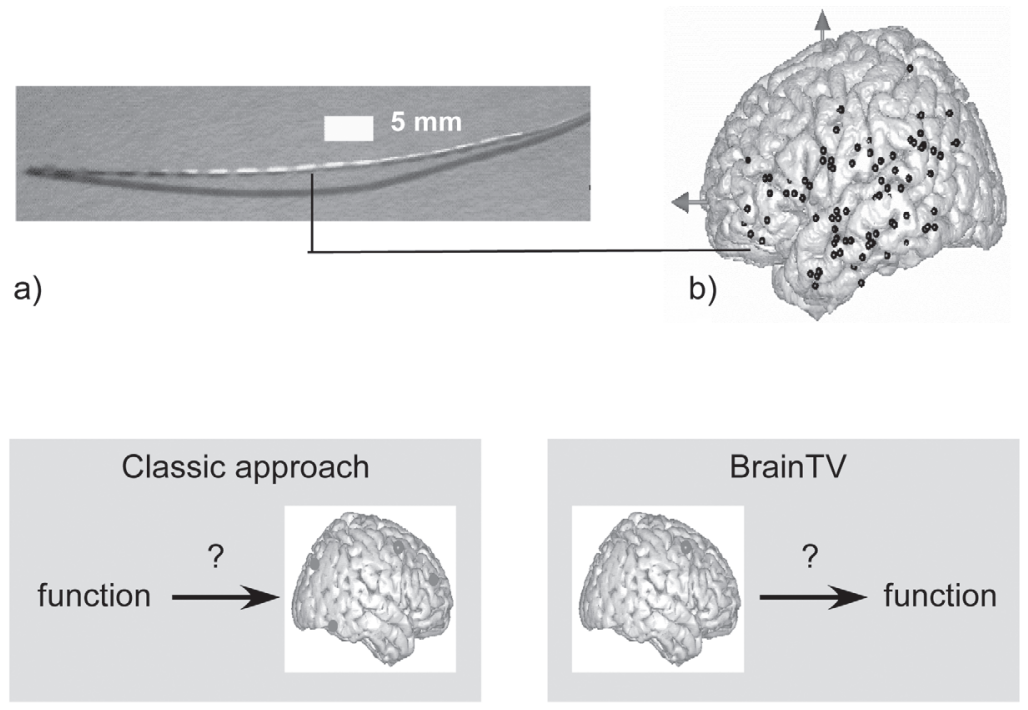

c)

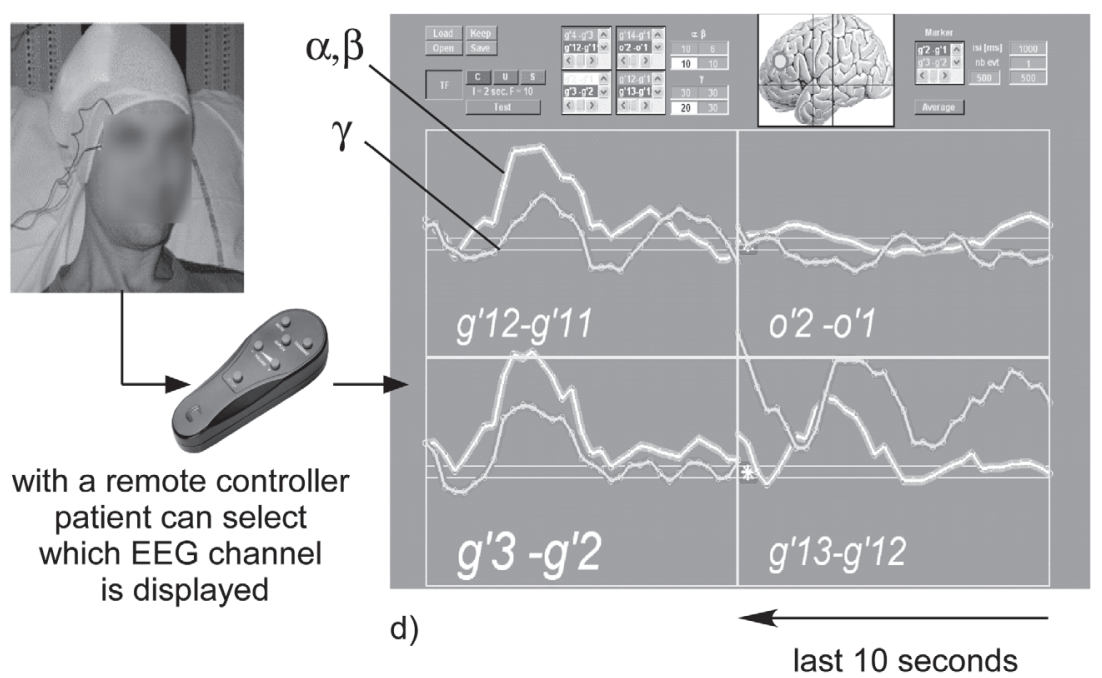

Figure 1: BrainTV setup. a) depth electrode used for intracranial EEG recordings in epileptic patients, it comprises 15 recording sites evenly spaced every $3.5 \mathrm{~mm}$; depth electrodes are orthogonal to the sagittal plane : typical entry points are shown as black dots on the 3D brain reconstruction (b). c) difference between the classical approach used in standard human brain mapping studies, and the brainTV approach. In the former, the goal is to find all brain regions associated with a predefined cognitive process; in the latter, the goal is to find all cognitive processes associated with a predefined brain region. d) BrainTV display : the patient is shown with a computer screen displaying, for two frequency bands $([8-30 \mathrm{~Hz}]$ in blue and [60-140 $\mathrm{Hz}]$ in orange) the energy measured in four cortical sites over the last 10 seconds (the four panels, with corresponding site names indicated in white). Displays are continuously updated and always show signals recorded in the last ten seconds. The energy is expressed in units of the standard deviation of the energy measured at this frequency in a reference baseline period. The display is on during the entire day and the patient decides when to watch and interact with it. Using a remote control, the patient can decide which part of his cortex is being visualized, literally surfing between recorded channels; a yellow dot on a simplified representation of the brain informs the patient of the anatomical origin of the visualized activity (upper part of the display). 
particular neural marker. Frequency bands displayed could be adjusted to match the electrophysiological signature of the recorded site. In this experiment, two frequency bands were chosen $([8-30 \mathrm{~Hz}]$ and $[60-140 \mathrm{~Hz}]$ ) to cover the alpha, beta and gamma bands while avoiding contamination by $50 \mathrm{~Hz}$ noise. Note that since alpha and beta bandwidths had been found to be extremely correlated in previous studies by our group, they were (additively) pooled together in one single curve. The software also allowed the experimenter to set activity display with a lag of 30 seconds. This allows for testing the validity of a patient's report that s/he could control his/her brain activity, as they sometimes misleadingly believe. The neurofeedback period consists of three sessions : a) a 'free search' session during which the patient is left alone with the functioning display which it can at his/her own leisure, observe and possibly notice systematic correlations with exogenous or endogenous events, b) a 'guided search' session, which, in case of failure of the free search, involves orienting the patient's attention to cognitive processes putatively effected by the cortical location under investigation. The 'guided search' session is based on the existing neuroimaging literature and on the results of previous cognitive experiments that the patient may have participated in. c) a 'confirmation' session, which follows the previous ones in case of success. The aim of this confirmation session is to test and refine any correlation found with the patient. Crucially, the success of the whole procedure depends on the patient's sustained motivation and interest.

\section{Experimental protocol}

Optimally, the confirmation session must be followed by a classic experimental session during which the patient performs a cognitive task centred on the cognitive processes identified through neurofeedback. This task can be either drawn from a (local) large bank of protocols (to date, for instance, twenty paradigms are ready-to-use in our center), or especially designed in the time remaining before the removal of the electrodes. Thus, neurofeedback sessions should ideally be conducted in the early days of the patient's implantation. The present experiment was designed to contrast six experimental conditions : a) patient listening to sentences spoken by the experimenter in his mother tongue (READ_EXP), b) patient reading aloud equivalent sentences in his mother tongue (READ_PAT), c) patient listening to sentences spoken by the experimenter in Finnish (Suomi), a language unknown both to him and the experimenter, (SUOM_EXP), d) patient listening to repeated nonsensical short speech patterns pronounced by the experimenter ('patapatapata...' or 'mumomumomumo....') (PATA_EXP), e) patient speaking self-generated sentences describing recently past events (TALK_PAT) and f) patient listening to drum patterns produced by the experimenter (NOIS_EXP). The protocol was block-designed, with 10 to 20 stimulus periods of about 10 seconds for each condition. Stimulus periods were separated by at least one second and a time-marker indicated the end of each stimulus period and was used for averaging.

\section{DATA ANALYSIS}

\section{Neurofeedback data analysis}

The neurofeedback program displayed the spectral energy of the signal of one particular bipolar derivation computed between adjacent electrode contacts. One cycle of analysis procedure consisted of a) applying a short-term Fourier transform to the signal (using a sliding Hamming window of 256 milliseconds moving by steps of $128 \mathrm{~ms}$ ) to extract the time-course of its spectral energy over the last 10 seconds for all frequencies between 1 and $150 \mathrm{~Hz}, \mathrm{~b})$ normalizing this time-frequency energy map (z-score) independently for each frequency, relative to the spectral energy measured during an earlier baseline period (while the patient was at rest, motionless, with his eyes opened in a quiet surrounding), c) averaging the TF energy in 
three frequency bands ([8-30 Hz], [60-90 $\mathrm{Hz}$, [110-140 Hz]), d) adding the [60-90 $\mathrm{Hz}]$ and [110 - $140 \mathrm{~Hz}]$ components (the aim is to cover the [60-140 Hz] frequency band while avoiding $50 \mathrm{~Hz}$ harmonics at $100 \mathrm{~Hz}, \mathrm{e})$ applying convolution in time with a two seconds time window, to increase the signal over noise ratio of the displayed signal and finally f) displaying those two 10 -second energy time courses in the form of two plots (figure 1; gamma shown in orange, alpha/beta in blue). The software was set to refresh the display as often as possible, that is, to get newly acquired data and analyze them as soon as the previous cycle of analysis-display had been completed. The program ran under Matlab (The Mathworks, inc) on a PC accessing the acquisition PC disk via an Ethernet connection. In effect, the refresh rate of the display is limited solely by the acquisition software (Micromed, Treviso, Italy) which writes new data on the acquisition hard drive every $250 \mathrm{~ms}$.

\section{Experimental protocol analysis}

For each single trial showing no sign of epileptiform activity, bipolar derivations computed between adjacent electrode contacts were analyzed in the timefrequency (TF) domain with a Morlet Wavelet transform, following our usual procedure (Mainy et al., 2007) as in the neurofeedback session. Investigated frequency range was [1-200 Hz] and the time range between $3000 \mathrm{~ms}$ before and $2000 \mathrm{~ms}$ after the time marker (set as the end of the stimulus period).

The analysis was designed to be as close as possible to that used during the neurofeedback session. For each trial, the TF energy map was normalized (z-score) independently for each frequency relative to the mean and standard deviation of a common baseline. More precisely, this normalization consisted in a) computing the mean and standard deviation of all the energy values measured during the [200 ms: $400 \mathrm{~ms}$ ] baseline in all the trials (for all the conditions), b) subtracting this mean energy from the TF map under process, and c) dividing by the standard deviation calculated above. Because the mean and the standard deviation were computed from baseline energy values pooled across all six experimental conditions, normalization was similar for all six maps. Given that during the neurofeedback session, the effects had been mostly observed in the [60-90 Hz] and $[110-140 \mathrm{~Hz}]$ ranges and with a $2000 \mathrm{~ms}$ averaging window, the statistical analysis compared across the six conditions the mean TF energy in the $([60-90 \mathrm{~Hz}]$ and [110-140 Hz]) x [-2000 ms : $0 \mathrm{~ms}])$ TF tile obtained in all the individual trials (i.e. the mean of energy values measured in both the [60-90 Hz] and the [110-140 Hz] ranges).

Statistical analysis of TF energy values was performed with a nonparametric Kruskal-Wallis test and Fisher's protected least significant differences based on ranks (FPLSD) for post hoc tests of significance (Portney and Watkins, 1993). The use of nonparametric tests was necessary because of the far from Gaussian distribution of TF energy values. It was performed on ranked data and provided a $\mathrm{H}$ value indicating whether a global effect of stimulation type was significant. If found significant, FPLSD tests allowed for comparisons of all the possible combinations of experimental condition pairs, and thus to determine in which pairs significant differences occurred.

For visualization purposes (Figure 2), the TF maps were averaged across all trials in a given experimental condition and normalized through the procedure described above.

\section{RESULTS}

\section{Neurofeedback session}

The neurofeedback consisted of presenting the patient with a display of his own brain activity (figure 1). In this case, imaged activity was presented for site A (not visible in the display in figure 1) in the mid part of the upper bank of the left superior temporal sulcus (mSTS) (Talairach coordinates: $-65,-10,5 \mathrm{~mm}$ ) (figure 2), together with three other sites. The display appeared as two dynamic curves displaying energy levels measured in two frequency 
bands $([8-30 \mathrm{~Hz}],[60-140 \mathrm{~Hz}]$ over the previous ten seconds.

The Neurofeedback session started with a free search period, during which we simply let the patient become familiar with variations of the display. The patient was informed that the display represented his cerebral activity in real time, but no information was given pertaining to the putative function of the cortical area. We encouraged the patient to be alert to possible temporal correlations between the
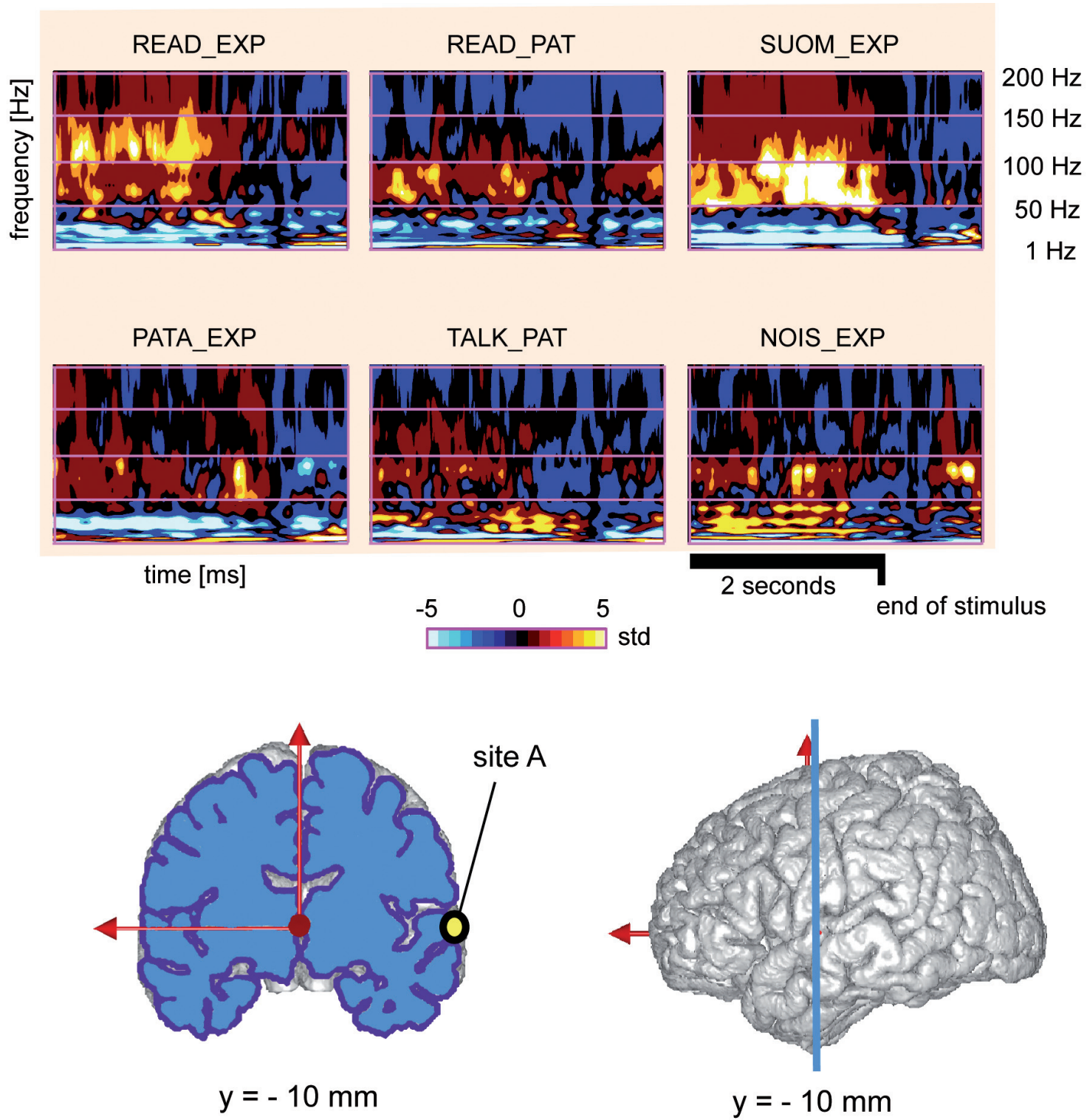

Figure 2: Spectral Energy variations associated with speech in the mid superior temporal sulcus (site A in this study). Each map represents for each frequency below $200 \mathrm{~Hz}$, the time course of spectral energy measured in site A (Talairach coordinates : -65, -10,5) over the last two seconds of auditory stimulation (black horizontal bar) and over the first second after. The six graphs correspond to the six experimental conditions. At each frequency the energy is expressed in units of the standard deviation of the energy measured at this frequency in the [200 ms : $400 \mathrm{~ms}$ ] period. The normalization parameters were the same for all maps. Recording site is represented on a 3D reconstruction of the Mni single-subject's brain template. 
marker and events in his environment or in his own experience, both at rest and during usual activities such as reading. The display representing the last 10 seconds of cortical activity, made it possible for the patient to switch his attention from time to time between the task at hand and the display to notice correlations. Site A was chosen because it had earlier evidenced, for this patient, a gamma-band energy increase to visually presented words and pseudo-words during a silent reading task. We therefore anticipated that, while reading his book, the patient might notice an increase of the marker displayed .

This was not evident to the patient. So, after 10 minutes of free search period, conducted in silence, the experimenter entered the room and asked the patient if he had noticed anything, eliciting a negative answer, including for reading. However, the conversation itself triggered a strong increase of the index, first interpreted as a general arousal effect, but which quickly turned out to be more specific. Three minutes of exchange between experimenter and patient followed, centred on the relationship between the marker and reading, during which both persons read aloud in turn. After this period, it was clear that the marker reacted selectively to the experimenter's speech The neurofeedback session went on for another thirty minutes, during which multiple members of the staff went in to try out multiple tests, such as repeating nonsense speech patterns ('patapatapata' : no response of the marker), or talking in multiple languages (spoken or not by the patient : response, sometimes stronger than for French, such as for German, which the patient understood at school level). At the end of this session, it was evident to the staff that the region was most strongly activated when the patient was attentive to spoken sentences, whether in known or unknown languages. In comparison, the region was not activated even by louder noises, such as drum patterns, or by the patient's own speech, predictable speech patterns such as the alphabet, or by loud conversations between third parties while the patient was talking to the experimenter.

\section{Experimental session}

The design of the experiment was adapted to the observations made during the neurofeedback session, and had the patient listen to sentences pronounced in his mother tongue (READ_EXP), in an unknown language (SUOM_EXP), or to non-sensical speech patterns (PATA_EXP) or loud noises (NOIS_EXP) or read sentences in French (READ_PAT) or simply talk (TALK_PAT). Figure 2 shows the time-frequency energy maps for these six conditions, normalized relative to a common baseline. The maps, which represent the energy averaged over the multiple trials of each condition, show a clear decrease in gamma band activity at sentence end in two conditions only (READ_EXP and SUOM_EXP).

To quantify this effect, we compared between conditions the energy recorded at site $A$ in the $[60-90 \mathrm{~Hz}]$ and [110 - 140 $\mathrm{Hz}$ ] ranges averaged over the last two seconds of each speech or noise period. This choice was motivated by the fact that the most efficient marker during the neurofeedback session had been the energy of the past two seconds in those ranges. Figure 2 shows the gamma band activation elicited during the six conditions. A Kruskal-Wallis analysis revealed a significant effect of the experimental condition $(\mathrm{H}=45.7$, $\mathrm{p}<0.0001)$. Post-hoc Fisher's protected least significant differences test applied on ranks showed that the strongest energy was obtained in the SUOM_EXP condition (SUOM_EXP > READ_PAT, PATA_EXP, TALK_PAT and NOIS_EXP, $p<0.05)$, the difference between the SUOM_EXP and the READ_EXP was not significant (in addition, we observed READ_EXP > NOIS_EXP, p<0.05). For information, Mann-Whitney comparisons between the READ_EXP and the other conditions indicated a difference with the READ_PAT, PATA_EXP and NOIS_EXP $(p<0.0 \overline{1})$. The same test was significant between the READ_EXP and the SUOM_EXP conditions $(\mathrm{p}<0.05)$, with SUOM_EXP $>$ READ_EXP. 


\section{Effects of Electric Cortical Stimulations}

A first session of ECS took place before the neurofeedback session and revealed no noticeable effects on site A, either at $1 \mathrm{~Hz}$ or at $50 \mathrm{~Hz}$ (up to $3 \mathrm{~mA}$ ) (the patient had to count aloud, or listen to the experimenter counting, 'did you hear me correctly ?'). However, the behaviour of this site during the neurofeedback led us to stimulate this site in a second session during which we tested specifically the patient for speech perception impairment, and this time, it was noticed that $50 \mathrm{~Hz}$ stimulations $(3 \mathrm{~mA})$ produced difficulties in sentence understanding.

\section{DISCUSSION}

The main outcome of this study is to demonstrate that gamma band energy modulations could be used as online markers of high-order cognitive processes in humans. To our knowledge, this observation has thus far only been reported for lower frequency activities or in the motor cortex and early sensory regions during simple movements or sensory stimulations (Chatrian et al., 1960, Graimann et al., 2004, Leuthardt et al., 2004, Pfurtscheller et al., 2006), that is, in regions whose functional organisation is already well-known and which can be readily identified using ECS. Our results demonstrate the validity of neurofeedback for the identification of certain eloquent brain areas in humans, in conjunction with standard ECS. Both techniques should be thought of as complementary: the neurofeedback led us to specify precise functions whose perturbation had to be tested during the ECS session, and ECS revealed the effects on those functions of local perturbations of neural activity. The idea is not to replace ECS by neurofeedback sessions, but to complement it for certain functions.

It should also be noted that, as in previous intracranial studies, it was in the gamma band that the correlation with the patient's behaviour proved to be tightest. Although the visual display also presented the activity in the alpha and beta bands, the patient spontaneously used the gamma activity as the more efficient marker. The cortical origin of those gamma band modulations was consistent with the wellknown implication of the mid superior temporal sulcus in speech perception. Several neuroimaging studies have clearly established that this region activates in situations contrasting vocal vs. non vocal sounds (Belin et al., 2002, Belin et al., 2000 ), or human vs. animal vocal sounds (Fecteau et al., 2004). Indeed, in an fMRI study comparing short vocal vs. non-vocal sounds (Belin et al., 2002), the maximum BOLD difference was found only a few millimetres away from recording site $\mathrm{A}$ in the Talairach space (Talairach: -62, -14,0). Also, a recent study (Cohen et al., 2004) has proposed that a region very close to our recording site (Talairach: -60, -8, -4) may constitute an auditory analog of the Visual Word Form Area. The effect we observed is thus fully compatible with the neuroimaging literature and not surprising $a$ posteriori, however, we believe that the exact behaviour of site A during the neurofeedback session could not have been known a priori, not only because of the interindividual variability, but also because the exact function of this region in speech perception is still a matter of debate (Scott and Johnsrude, 2003). Several studies have proposed that it may be involved in sentence processing (Friederici et al., 2003, Humphries et al., 2005), either for the syntactic or for the semantic analysis of sentences (Friederici et al., 2003). In the patient we examined, this region responded strongly and selectively to sentences pronounced by the experimenter (in French or in an unknown foreign language), and less by the patient, and did not respond to non-sentence-like speech patterns such as the alphabet. This pattern of responses is very consistent with a participation in highlevel phonological decoding, which would explain why there was no response to the subject's own voice, because the patient did not decode his own sentences. However, it is unlikely that the region participated in semantic processing because the strongest response was obtained for sentences in 
Suomi, a language unknown to the patient. Interestingly, the activity in this region has been found to be altered in autistic children (Boddaert et al., 2004), which may explain certain difficulties in communicating with others. In our sense, those results show that the neurofeedback session actually brought new insights into the function of the mid STS during speech perception, and not a simple confirmation of the known functional anatomy of this region.

Altogether, our results support the use of neurofeedback for exploratory purposes, during functional cortical mapping. Every brain mapping study is based on the exploration of a 'stimulus-task' space, achieved through manipulations of both the environment of the subject and the task s/he is instructed to perform. Cognitive protocols rely on a parameterization of a subregion of that space, which then allows for correlating variations of brain activity measurements with variations of those parameters. Because cognitive experiments have to be relatively short, there is always a compromise between the size of the subregion explored and the degree of precision with which it is explored (a study on face perception can test the cerebral sensitivity to subtle differences in face shape, but does not reveal anything about the response to landscapes).

The approach that we propose is an attempt to overcome this constraint via a multiscale and stepwise exploration of the 'stimulus-task' space. The initial neurofeedback session allows for coarse exploration of very large portions of that space. Exploration then narrows down to much more specific and 'efficient' experimental manipulations eventually yielding a precisely controlled cognitive protocol. In fact, this approach is parallel to the mapping of receptive fields in $\mathrm{V} 1$, and can be thought of as a 'cognitive field mapping' procedure. Note that the initial neurofeedback exploration may be generally sufficient to convince both experimenter and patient of the functional role of the investigated area. Therefore, the use of precisely controlled protocols and precise statistical evaluations may not be mandatory in standard clinical practice, but only necessary for research purposes.
An important methodological question is whether the initial exploration should be guided or whether the patient should be left alone with the feedback display on, to try without help to find correlates of his/her behaviour and experience. The answer largely depends on the time available for exploration, and on the patient ability for introspection. For a quicker search, it may be helpful to encourage the patient specifically to explore certain cognitive processes in particular, based on the general knowledge gained from functional neuroimaging of the cortical area under investigation. In this regard, one should note that recent studies have shown a clear correlation between the recordings of BOLD signal and gamma activity (Kim et al., 2004, Lachaux et al., in press, Mukamel et al., 2005, Niessing et al., 2005), which suggest that the fMRI literature can be used to narrow down the patient's exploration to specific cognitive processes. However, the free search phase potentially allows to test for effects not shown yet with fMRI, and also to deal with possible cortical reorganisations specific to an individual.

A further question is whether this approach can be used for all structures and functions. It is well known to work efficiently for simple sensory and motor processes, and our results demonstrate its validity for high-order auditory processes, but it is not clear yet whether it is adapted for more endogenous mental events, such as slight emotional or attentional modulations. The limits of the approach are in fact determined by two factors a) the introspective capacity of the patient to identify in his/her own subjective experience events correlated in time with the feedback neural marker and b) the intrinsic variability of the neural marker.

The first point is at the center of a larger research project known as 'neurophenomenology' (Petitot et al., 1999, Thompson and Varela, 2001, Varela and Shear, 1999, Varela, 1996) which explores the advantages and limits of combining 'first-person data' (subjective reports) with 'third-person' neuroimaging data. It is enough here to say that this project poses methodological challenges, which have 
been addressed by several authors (Varela and Shear, 1999). We will simply note here that the procedure can greatly benefit from the assistance of an expert in guiding the patient's introspection (Petitmengin, 2006). At the very least, it is fair to say that the precision of information the patient can provide is directly influenced by the time s/ he has had to familiarize herself/himself with her/his displayed neural activity. Note that the different site markers need not to be shown in turn, one by one, as in the present experiment (although this allows both the patient and the experimenter to focus on one single site), but one may envision to display continuously the activity of several sites simultaneously (visually, or through sonification) and let the patient become familiar with them at leisure. Another point raised by the neurophenomenological approach is whether the experimenter can validly trust patients reports (Jack and Roepstorff, 2004), which is a real issue when the neural marker correlates with mental events accessible only to the patient. At some point the experimenter has to trust the subjective report of the patient (as with ECS mapping). What can be tested objectively is whether the patient actually found a correlation between the objective and a subjective marker, because s/he must then be able to signal the variations of the objective marker based only on her/his own experience even if he has no access to the feedback. Also, s/he must be able to identify catch trials in which the activity that is fed back is not real-time, but recorded say a minute earlier. Furthermore, once a subjective event is clearly recognised by the subject, s/he might still not be able adequately to verbalise it, or might yet succeed in fooling the experimenter. These considerations further militate for the careful design of protocols to manipulate specifically the dimensions reported by the patient to validate her/his description.

The second limitation of the neurofeedback approach comes from the variability of neural signals. Even if precautions are taken to avoid variations in the subject's environment, there is a high degree of variability in neural responses even to identical external stimulations (Arieli et al., 1996). This variability is more pronounced in recordings with a low spatial resolution, such as scalp EEG, than with more precise recordings such as intracranial EEG, which suggests that it is partly due to the contamination of the signal by neural activities not related to the repeated experimental process. For this reason, all the studies reporting task-related gamma band modulations have had to rely on averaging procedures across multiple repetitions of the same situation. This observed variability is lower in early sensory areas or in the motor cortex. For instance, the activity that can be recorded in the primary auditory cortex in human with intracranial EEG electrodes faithfully reflects the envelope of the auditory stimulus, with little noise (LiegeoisChauvel et al., 2004). However, variability increases in more associative brain regions, which may limit the interpretability of the signal by the patient in those areas. The critical point is whether or not this variability leaves a trace in the patient's conscious experience. It has been shown that some of the spontaneous fluctuations are due to the modulation of actual cognitive processes that are usually left uncontrolled, such as attentional and emotional fluctuations or random thoughts, of which the patient can become aware of (Lutz et al., 2002). For such sources of variability, it is likely that the patients could quickly learn to control and stabilize the variability in neural activity. This would predict that the variability of the marker should decrease with practice.

In conclusion, this study demonstrates that neurofeedback sessions based on online Dynamic Spectral Imaging can improve the identification of eloquent brain areas in epileptic patients candidate for surgery. Further, these sessions provide a powerful way of understanding the anatomical basis of cognition when used in conjunction with well-calibrated cognitive paradigms. The recent development of new source reconstruction algorithms should enable online Dynamical Spectral Imaging with high spatial resolution also in normal subjects 
recorded non-invasively with MEG (Hoogenboom et al., 2006). This paves the way for rehabilitation therapy techniques that would provide feedback to the subjects' neural markers of specific cognitive processes, such as attention or memory, to be trained or retrained (De Charms et al., 2005).

\section{ACKNOWLEDGEMENTS}

We thank Valérie Balle, Patricia Boschetti, Carole Chatelard, Véronique Dorlin, Eliane Gamblin and Martine Juillard for their invaluable help. We specially thank Danielle David for help with the experimental procedure and Rainer Goebel for helpful comments on an earlier version of the manuscript. This work is dedicated to the late Francisco Varela (1945-2001).

\section{REFERENCES}

ARIELI A, STERKIN A, GRINVALD A, AERTSEN A (1996) Dynamics of ongoing activity: explanation of the large variability in evoked cortical responses. Science 273: 1868-1871

BELIN P, ZATORRE RJ, AHAD P (2002) Human temporal-lobe response to vocal sounds. Brain Res Cogn Brain Res 13: 17-26

BELIN P, ZATORRE RJ, LAFAILLE P, AHAD P, PIKE B (2000) Voice-selective areas in human auditory cortex. Nature 403: 309-312

BIDET-CAULET A, FISCHER C, BAUCHET F, BERTRAND O (2003) Multiple sources of induced gamma oscillations in the auditory cortex observed from human intracranial EEG. Neuroimage 19: 1554

BODDAERT N, CHABANE N, GERVAIS H, GOOD CD, BOURGEOIS M, PLUMET MH, BARTHELEMY C, MOUREN MC, ARTIGES E, SAMSON Y, BRUNELLE F, FRACKOWIAK RS, ZILBOVICIUS M (2004) Superior temporal sulcus anatomical abnormalities in childhood autism: a voxel-based morphometry MRI study. Neuroimage 23: 364-369

BROVELLI A, LACHAUX JP, KAHANE P, BOUSSAOUD D (2005) High gamma frequency oscillatory activity dissociates attention from intention in the human premotor cortex. Neuroimage 28: 154164

CHATRIAN GE, BICKFORD RG, UIHLEIN A (1960) Depth electrographic study of a fast rhythm evoked from the human calcarin region by steady illumination. Elec Clin Neurophysiol 12: 167-176

Cohen L, Jobert A, Le Bihan D, Dehaene S (2004) DISTINCT unimodal and multimodal regions for word processing in the left temporal cortex. Neuroimage 23: $1256-1270$

CRONE NE, BOATMAN D, GORDON B, HAO L (2001) Induced electrocorticographic gamma activity during auditory perception. Brazier Award-winning article, 2001. Clin Neurophysiol 112: 565-582
DE CHARMS RC, MAEDA F, GLOVER GH, LUDLOW D, PAULY JM, SONEJI D, GABRIELI JD, MACKEY SC (2005) Control over brain activation and pain learned by using real-time functional MRI. Proc Natl Acad Sci U S A 102: 18626-18631

DI PELLEGRINO G, FADIGA L, FOGASSI L, GALLESE V, RIZZOLATTI G (1992) Understanding motor events: a neurophysiological study. Exp Brain Res 91: 176-180

EDWARDS E, SOLTANI M, DEOUELL LY, BERGER MS, KNIGHT RT (2005) High gamma activity in response to deviant auditory stimuli recorded directly from human cortex. J Neurophysiol 94: 4269-4280

FECTEAU S, ARMONY JL, JOANETTE Y, BELIN P (2004) Is voice processing species-specific in human auditory cortex? An fMRI study. Neuroimage 23: 840848

FELL J, KLAVER P, LEHNERTZ K, GRUNWALD T, SCHALLER C, ELGER CE, FERNANDEZ G (2001) Human memory formation is accompanied by rhinalhippocampal coupling and decoupling. Nat Neurosci 4: 1259-1264

FRIEDERICI AD, RUSCHEMEYER SA, HAHNE A, FIEBACH CJ (2003) The role of left inferior frontal and superior temporal cortex in sentence comprehension: localizing syntactic and semantic processes. Cereb Cortex 13: 170-177

GRAIMANN B, HUGGINS JE, LEVINE SP PFURTSCHELLER G (2004) Toward a direct brain interface based on human subdural recordings and wavelet-packet analysis. IEEE Trans Biomed Eng 51: 954-962

HOOGENBOOM N, SCHOFFELEN JM, OOSTENVELD R, PARKES LM, FRIES P (2006) Localizing human visual gamma-band activity in frequency, time and space. Neuroimage 29: 764-773

HOWARD MW, RIZZUTO DS, CAPLAN JB, MADSEN JR, LISMAN J, ASCHENBRENNER-SCHEIBE R, SCHULZE-BONHAGE A, KAHANA MJ (2003) Gamma oscillations correlate with working memory load in humans. Cereb Cortex 13: 1369-1374

HUMPHRIES C, LOVE T, SWINNEY D, HICKOK G (2005) Response of anterior temporal cortex to syntactic and prosodic manipulations during sentence processing. Hum Brain Mapp 26: 128-138

JACK A, ROEPSTORFF A, eds. 2004. Trusting the subject ? The Use of Introspective Evidence in Cognitive Science. London: Imprint Academic

KAHANE P, HOFFMANN D, MINOTTI L, BERTHOZ A (2003) Reappraisal of the human vestibular cortex by cortical electrical stimulation study. Ann Neurol 54: 615-624

KAHANE P, MINOTTI L, HOFFMANN D, LACHAUX J, RYVLIN P (2004) Invasive EEG in the definition of the seizure onset zone: depth electrodes. In: Handbook of Clinical Neurophysiology. Pre-surgical assessment of the epilepsies with clinical neurophysiology and functional neuroimaging (Rosenow, F. and Lüders, H.O., eds.): Elsevier Science

KAHANE P, TASSI L, FRANCIONE S, HOFFMANN D, LO RUSSO G, MUNARI C (1993) Manifestations électro-cliniques induites par la stimulation électrique intra-cérébrale par "chocs" dans les épilepsies temporales. Neurophysiol Clin 22: 305-326

KIM DS, RONEN I, OLMAN C, KIM SG, UGURBIL K, TOTH LJ (2004) Spatial relationship between neuronal activity and BOLD functional MRI. Neuroimage 21: 876-885

LACHAUX JP, FONLUPT P, KAHANE P, MINOTTI L, HOFFMANN D, BERTRAND O, BACIU M (in press) 
On the relationship between task-related Gamma oscillations and BOLD signal: new insights from combined fMRI and intracranial EEG. Human Brain Mapping

LACHAUX JP, HOFFMANN D, MINOTTI L, BERTHOZ A, KAHANE P (2005) Intracerebral Dynamics of Saccade Generation in the Human Frontal Eye Field and Supplementary Eye Field. Neuroimage in press

LACHAUX JP, RODRIGUEZ E, MARTINERIE J, ADAM C, HASBOUN D, VARELA FJ (2000) A quantitative study of gamma-band activity in human intracranial recordings triggered by visual stimuli. Eur J Neurosci 12: $2608-2622$

LACHAUX JP, RUDRAUF D, KAHANE P (2003) Intracranial EEG and human brain mapping. J Physiol Paris 97: 613-628

LESSER RP, ARROYO S, CRONE N, GORDON B (1998) Motor and sensory mapping of the frontal and occipital lobes. Epilepsia 39 Suppl 4: S69-80

LEUTHARDT EC, SCHALK G, WOLPAW JR, OJEMANN JG, MORAN DW (2004) A brain-computer interface using electrocorticographic signals in humans. J Neural Eng 1: 63-71

LIEGEOIS-CHAUVEL C, LORENZI C, TREBUCHON A, REGIS J, CHAUVEL P (2004) Temporal envelope processing in the human left and right auditory cortices. Cereb Cortex 14: 731-740

LOBEL E, KAHANE P, LEONARDS U, GROSBRAS M, LEHERICY S, LE BIHAN D, BERTHOZ A (2001) Localization of human frontal eye fields: anatomical and functional findings of functional magnetic resonance imaging and intracerebral electrical stimulation. J Neurosurg 95: 804-815

LUDERS HO, DINNER DS, MORRIS HH, WYLLIE E, COMAIR YG (1995) Cortical electrical stimulation in humans. The negative motor areas. Adv Neurol 67: 115-129

LUTZ A, LACHAUX JP, MARTINERIE J, VARELA FJ (2002) Guiding the study of brain dynamics by using first-person data: synchrony patterns correlate with ongoing conscious states during a simple visual task. Proc Natl Acad Sci U S A 99: 1586-1591

MAINY N, KAHANE P, MINOTTI L, HOFFMANN D, BERTRAND O, LACHAUX JP (2007) Neural correlates of consolidation in working memory. Hum Brain Mapp 28: 183-193

MUKAMEL R, GELBARD H, ARIELI A, HASSON U, FRIED I, MALACH R (2005) Coupling between neuronal firing, field potentials, and FMRI in human auditory cortex. Science 309: 951-954

NIESSING J, EBISCH B, SCHMIDT KE, NIESSING M, SINGER W, GALUSKE RA (2005) Hemodynamic signals correlate tightly with synchronized gamma oscillations. Science 309: 948-951

OJEMANN G, OJEMANN J, LETTICH E, BERGER M (1989) Cortical language localization in left, dominant hemisphere. An electrical stimulation mapping investigation in 117 patients. J Neurosurg 71: $316-326$

PENFIELD W, JASPER H (1954) Epilepsy and the functional anatomy of the human brain. Boston: Little Brown
PETITMENGIN C (2006) Describing one's subjective experience in the second-person. Phenomenology and the Cognitive Sciences (in press)

PETITOT J, VARELA FJ, PACHOUD B, ROY JM, eds. 1999. Naturalizing Phenomenology: Contemporary Issues in Phenomenology and Cognitive Science. Stanford: Stanford University Press

PFURTSCHELLER G, BRUNNER C, SCHLOGL A, LOPES DA SILVA FH (2006) Mu rhythm (de)synchronization and EEG single-trial classification of different motor imagery tasks. Neuroimage

PFURTSCHELLER G, GRAIMANN B, HUGGINS JE, LEVINE SP, SCHUH LA (2003) Spatiotemporal patterns of beta desynchronization and gamma synchronization in corticographic data during selfpaced movement. Clin Neurophysiol 114: 1226-1236

PORTNEY LG, WATKINS MP (1993) In: Foundations of Clinical Research Applications and practice, pp 430432. London: Prentice Hall International

SCOTT SK, JOHNSRUDE IS (2003) The neuroanatomical and functional organization of speech perception. Trends Neurosci 26: 100-107

SINAI A, BOWERS CW, CRAINICEANU CM, BOATMAN D, GORDON B, LESSER RP, LENZ FA, CRONE NE (2005) Electrocorticographic high gamma activity versus electrical cortical stimulation mapping of naming. Brain 128: 1556-1570

SINGER W (1999) Neuronal synchrony: a versatile code for the definition of relations? Neuron 24: 49-65, 111125

SZURHAJ W, BOURRIEZ JL, KAHANE P, CHAUVEL P, MAUGUIERE F, DERAMBURE P (2005) Intracerebral study of gamma rhythm reactivity in the sensorimotor cortex. Eur J Neurosci 21: 1223-1235

TALAIRACH J, TOURNOUX P (1988) Co-planar stereotaxic atlas of the human brain. 3-dimensional proportional system: an approach to cerebral imaging. New York: Thieme

TALLON-BAUDRY C, BERTRAND O (1999) Oscillatory gamma activity in humans and its role in object representation. Trends Cogn Sci 3: 151-162

TANJI K, SUZUKI K, DELORME A, SHAMOTO H, NAKASATO N (2005) High-frequency gamma-band activity in the basal temporal cortex during picturenaming and lexical-decision tasks. J Neurosci 25: 3287-3293

THOMPSON E, VARELA FJ (2001) Radical embodiment: neural dynamics and consciousness. Trends Cogn Sci 5: 418-425

VARELA F, SHEAR J, eds. 1999. The View from Within: First-Person Methodologies in the Study of Consciousness. London: Imprint Academic

VARELA FJ (1996) Neurophenomenology: a methodological remedy for the hard problem. Journal of Consciousness Studies, 3: 330-350

VAUGHAN TM, HEETDERKS WJ, TREJO LJ, RYMER WZ, WEINRICH M, MOORE MM, KUBLER A, DOBKIN BH, BIRBAUMER N, DONCHIN E, WOLPAW EW, WOLPAW JR (2003) Brain-computer interface technology: a review of the Second International Meeting. IEEE Trans Neural Syst Rehabil Eng 11: 94-109 
